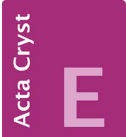

CRYSTALLOGRAPHIC COMMUNICATIONS

ISSN 2056-9890

\section{Crystal structure of 3-ethynylbenzoic acid}

\section{Chiara Venturini, Nicolas Ratel-Ramond and Andre Gourdon*}

CEMES-CNRS BP 94347, 29 Rue J. Marvig, 31055 Toulouse, France. *Correspondence e-mail: andre.gourdon@cemes.fr

Received 26 August 2015; accepted 4 September 2015

Edited by H. Stoeckli-Evans, University of Neuchâtel, Switzerland

In the title compound, $\mathrm{C}_{9} \mathrm{H}_{6} \mathrm{O}_{2}$, the carboxylic acid group is almost in the plane of the benzene ring, making a dihedral angle of $2.49(18)^{\circ}$. In the crystal, molecules are linked by pairs of $\mathrm{O}-\mathrm{H} \cdots \mathrm{O}$ hydrogen bonds, forming classical acid-acid inversion dimers, with an $R_{2}^{2}(8)$ ring motif. The dimers are linked by pairs of $\mathrm{C}-\mathrm{H} \cdots \mathrm{O}$ hydrogen bonds forming chains, enclosing $R_{2}^{2}(16)$ ring motifs, propagating along the $c$-axis direction.

Keywords: crystal structure; 3-ethynylbenzoic acid; hydrogen bonding.

CCDC reference: 1422308

\section{Related literature}

For the potential applications of terminal alkynes in crystal engineering, see: Dai et al. (2004). For the synthesis of the title compound, see: Bischoff et al. (2008). For the NMR spectrum of the title compound, see: Bleisch et al. (2014). For other syntheses of the title compound, see: Jones et al. (2008); Pawle et al. (2011). For the crystal structure of the 4-ethynyl benzoic acid methyl ester, see: Dai et al. (2004).

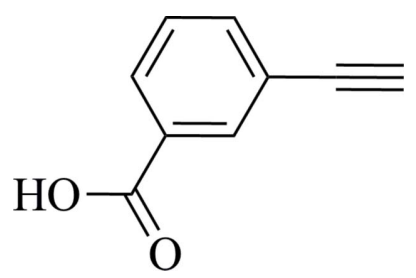

\section{Experimental}

\subsection{Crystal data}

$\mathrm{C}_{9} \mathrm{H}_{6} \mathrm{O}_{2} \quad$ Triclinic, $P \overline{1}$

$M_{r}=146.14 \quad a=3.8630$ (7) $\AA$

$$
\begin{aligned}
& b=8.3000(9) \AA \\
& c=11.7490(1) \AA \\
& \alpha=101.44^{\circ} \\
& \beta=93.8^{\circ} \\
& \gamma=99.83^{\circ} \\
& V=361.84(8) \AA^{3}
\end{aligned}
$$

\subsection{Data collection}

Bruker Kappa APEXII CCD diffractometer

Absorption correction: multi-scan (SADABS; Bruker, 2012)

$T_{\min }=0.664, T_{\max }=0.745$

\subsection{Refinement}

$R\left[F^{2}>2 \sigma\left(F^{2}\right)\right]=0.037$

$w R\left(F^{2}\right)=0.117$

$S=1.08$

1317 reflections
$Z=2$

Mo $K \alpha$ radiation

$\mu=0.10 \mathrm{~mm}^{-1}$

$T=293 \mathrm{~K}$

$0.9 \times 0.4 \times 0.1 \mathrm{~mm}$

Table 1

Hydrogen-bond geometry $\left(\AA,^{\circ}\right)$.

\begin{tabular}{lllll}
\hline$D-\mathrm{H} \cdots A$ & $D-\mathrm{H}$ & $\mathrm{H} \cdots A$ & $D \cdots A$ & $D-\mathrm{H} \cdots A$ \\
\hline $\mathrm{O} 1-\mathrm{H} 1 \cdots \mathrm{O} 2{ }^{\mathrm{i}}$ & $1.03(2)$ & $1.59(2)$ & $2.625(2)$ & $175(2)$ \\
$\mathrm{C} 9-\mathrm{H} 9 \cdots \mathrm{O} 2^{\mathrm{ii}}$ & $0.96(2)$ & $2.50(2)$ & $3.386(2)$ & $153(2)$ \\
\hline
\end{tabular}

Symmetry codes: (i) $-x+1,-y,-z+2$; (ii) $-x+1,-y,-z+1$.

Data collection: APEX2 (Bruker, 2012); cell refinement: SAINT (Bruker, 2012); data reduction: $S A I N T$; program(s) used to solve structure: SIR2011 (Burla et al., 2012); program(s) used to refine structure: SHELXL97 (Sheldrick, 2008); molecular graphics: Mercury (Macrae et al., 2008); software used to prepare material for publication: WinGX (Farrugia, 2012) and PLATON (Spek, 2009).

\title{
Acknowledgements
}

Support from the ANR-DFG project ICMADS is gratefully acknowledged.

Supporting information for this paper is available from the IUCr electronic archives (Reference: SU5200).

\section{References}

Bischoff, A., Subramanya, H., Sundaresan, K., Sammeta, S. \& Vaka (2008). Espacenet Patent WO2008157844 (A1).

Bleisch, T. J., Doti, R. A., Pfeifer, L. A. \& Norman, B. H. (2014). Espacenet Patent WO2014168824 (A1).

Bruker (2012). APEX2, SAINT and SADABS. Bruker AXS Inc, Madison, Wisconsin, USA.

Burla, M. C., Caliandro, R., Camalli, M., Carrozzini, B., Cascarano, G. L., Giacovazzo, C., Mallamo, M., Mazzone, A., Polidori, G. \& Spagna, R. (2012). J. Appl. Cryst. 45, 357-361.

Dai, C., Yuan, Z., Collings, J. C., Fasina, T. M., Thomas, R. Ll., Roscoe, K. P., Stimson, L. M., Batsanov, A. S., Howard, J. A. K. \& Marder, T. B. (2004), CrystEngComm, 6, 184-188.

Farrugia, L. J. (2012). J. Appl. Cryst. 45, 849-854.

Jones, L. F., Cochrane, M. E., Koivisto, B. D., Leigh, D. A., Perlepes, S. P., Wernsdorfer, W. \& Brechin, E. K. (2008). Inorg. Chim. Acta, 361, 34203426.

Macrae, C. F., Bruno, I. J., Chisholm, J. A., Edgington, P. R., McCabe, P., Pidcock, E., Rodriguez-Monge, L., Taylor, R., van de Streek, J. \& Wood, P. A. (2008). J. Appl. Cryst. 41, 466-470. 
Pawle, R. H., Eastman, V. \& Thomas, S. W. (2011). J. Mater. Chem. 21, 1404114047.
Sheldrick, G. M. (2008). Acta Cryst. A64, 112-122.

Spek, A. L. (2009). Acta Cryst. D65, 148-155. 


\section{supporting information}

Acta Cryst. (2015). E71, o750-o751 [doi:10.1107/S2056989015016515]

\section{Crystal structure of 3-ethynylbenzoic acid}

\section{Chiara Venturini, Nicolas Ratel-Ramond and Andre Gourdon}

\section{S1. Chemical context}

In recent years, the interest in compounds with an alkyne $\mathrm{C} \equiv \mathrm{CH}$ bond has increased due to their versatility in coupling reactions such as Glaser-Hay or Sonogashira. At the same time the crystallography of terminal alkynes has become an intense field of study for the potential applications in crystal engineering (Dai et al., 2004). Additionally, the presence of a carboxylate group on these compounds makes them potential candidates for the formation of metal organic frameworks viz. MOFs. With these applications in mind, we have synthesized the title compound and report herein on its crystal structure.The synthesis of 4-ethynylbenzoic acid has been reported previously (Jones et al., 2008; Pawle et al., 2011), but not its crystal structure. Only the crystal structure of the ester, 4-ethynylmethylbenzoate, has been described previously (Dai et al., 2004).

\section{S2. Synthesis and crystallization}

3-ethynylbenzoic acid is commercially available. In this work it was obtained by saponification and acidification of the corresponding ester in methanol/water with lithium hydroxide, following the reported procedure (Bischoff et al., 2008). Vapor diffusion of dichlomethane/hexane afforded light yellow crystals. The NMR spectrum is in agreement with the literature values (Bleisch et al., 2014): ${ }^{1} \mathrm{H}-\mathrm{NMR}(300 \mathrm{MHz}, \mathrm{DMSO}) 1 \mathrm{H} 13.22(1 \mathrm{H}, \mathrm{s}, \mathrm{O}-\mathrm{H}), 7.94-7.97$ (2H, m, H6; H2), 7.73-7.71 (1H, m, H3), 7.53 (1H, t, J = 7.5 Hz, H7), 4.30 (1H, s, CHh). ${ }^{13} \mathrm{C}-\mathrm{NMR}$ (75 MHz, DMSO) 13C 166.4 (Ci), $135.8(\mathrm{Cd}), 132.2(\mathrm{Ca}), 131.3(\mathrm{Ch}), 129.7(\mathrm{Cb}), 129.2(\mathrm{Cc}), 122.1(\mathrm{Ce}), 82.5(\mathrm{Cf}), 81.69(\mathrm{Cg})$.

\section{S2.1. Refinement}

Crystal data, data collection and structure refinement details are summarized in Table 2. All of the $\mathrm{H}$ atoms were located in difference Fourier maps and freely refined. 


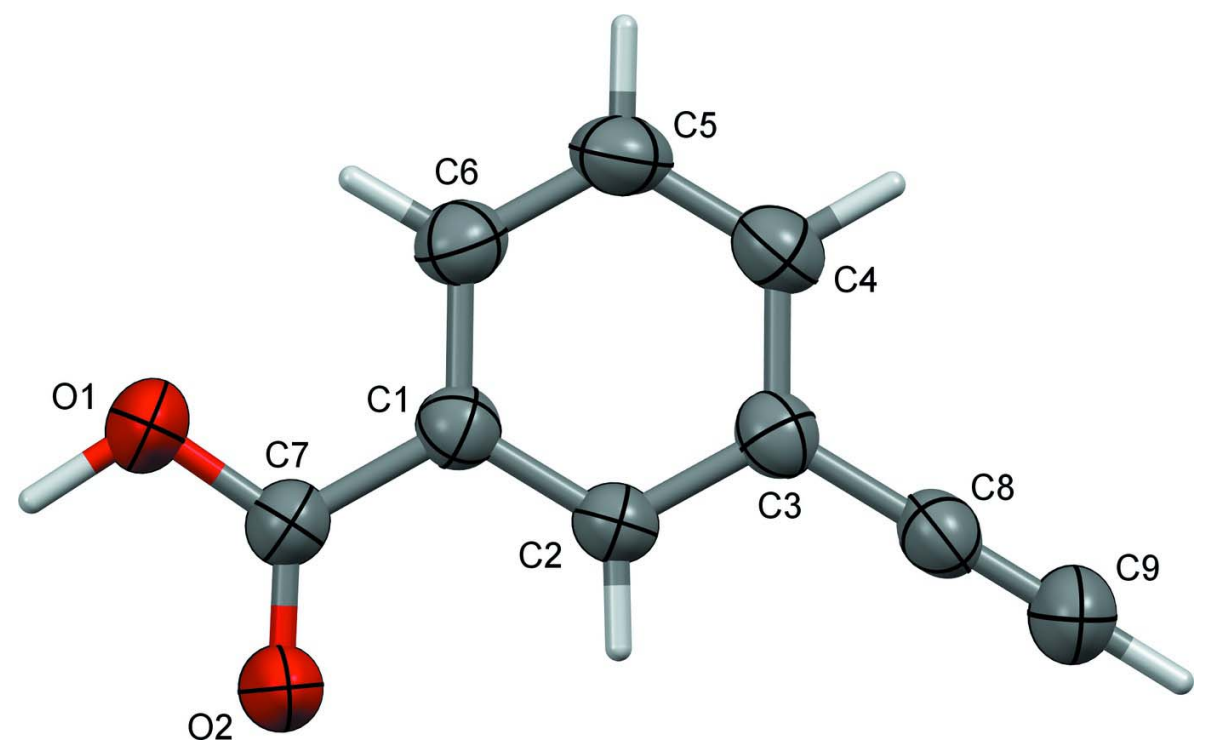

\section{Figure 1}

The molecular structure of the title compound, with atom labelling. Displacement ellipsoids are drawn at the 50\% probability level.

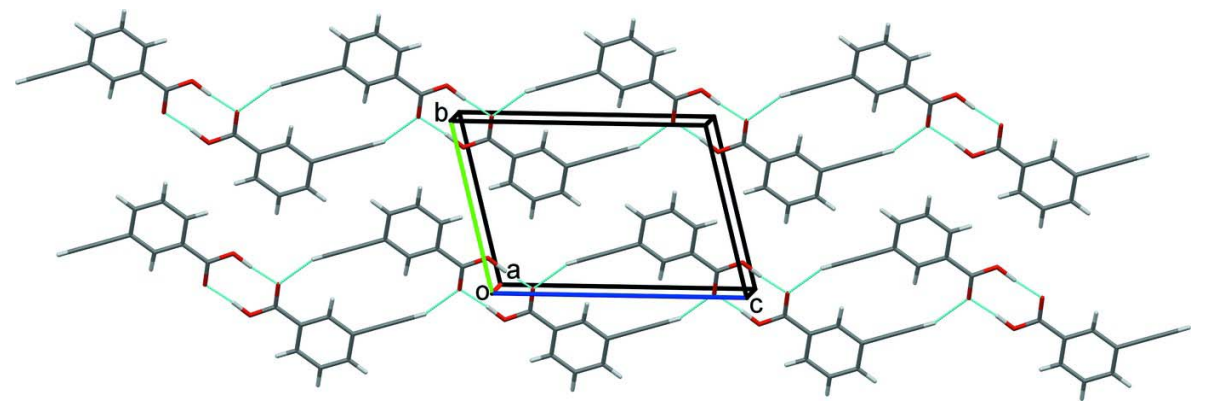

Figure 2

A view along the $a$ axis of the crystal packing of the title compound. The hydrogen bonds are shown as dashed lines (see

Table 1).

\section{3-Ethynylbenzoic acid}

Crystal data

$\mathrm{C}_{9} \mathrm{H}_{6} \mathrm{O}_{2}$

$M_{r}=146.14$

Triclinic, $P \overline{1}$

Hall symbol: -P 1

$a=3.8630$ (7) $\AA$

$b=8.3000$ (9) $\AA$

$c=11.7490(1) \AA$

$\alpha=101.44^{\circ}$

$\beta=93.8^{\circ}$

$\gamma=99.83^{\circ}$

$V=361.84(8) \AA^{3}$
$Z=2$

$F(000)=152$

$D_{\mathrm{x}}=1.341 \mathrm{Mg} \mathrm{m}^{-3}$

Mo $K \alpha$ radiation, $\lambda=0.71073 \AA$

Cell parameters from 1127 reflections

$\theta=2.6-26.1^{\circ}$

$\mu=0.10 \mathrm{~mm}^{-1}$

$T=293 \mathrm{~K}$

Parallelepiped, colourless

$0.9 \times 0.4 \times 0.1 \mathrm{~mm}$ 


\section{Data collection}

Bruker Kappa APEXII CCD diffractometer

Radiation source: sealed tube Graphite monochromator

phi and $\omega$ scans

Absorption correction: multi-scan (SADABS; Bruker, 2012)

$T_{\min }=0.664, T_{\max }=0.745$

\section{Refinement}

Refinement on $F^{2}$

Least-squares matrix: full

$R\left[F^{2}>2 \sigma\left(F^{2}\right)\right]=0.037$

$w R\left(F^{2}\right)=0.117$

$S=1.08$

1317 reflections

124 parameters

0 restraints
2719 measured reflections

1317 independent reflections

1086 reflections with $I>2 \sigma(I)$

$R_{\text {int }}=0.012$

$\theta_{\text {max }}=26.2^{\circ}, \theta_{\text {min }}=3.4^{\circ}$

$h=-3 \rightarrow 4$

$k=-10 \rightarrow 10$

$l=-14 \rightarrow 14$

0 constraints

Hydrogen site location: difference Fourier map

All $\mathrm{H}$-atom parameters refined

$w=1 /\left[\sigma^{2}\left(F_{\mathrm{o}}^{2}\right)+(0.0649 P)^{2}+0.035 P\right]$

where $P=\left(F_{\mathrm{o}}^{2}+2 F_{\mathrm{c}}^{2}\right) / 3$

$(\Delta / \sigma)_{\max }<0.001$

$\Delta \rho_{\max }=0.13$ e $\AA^{-3}$

$\Delta \rho_{\min }=-0.13$ e $\AA^{-3}$

Special details

Geometry. Bond distances, angles etc. have been calculated using the rounded fractional coordinates. All su's are estimated from the variances of the (full) variance-covariance matrix. The cell esds are taken into account in the estimation of distances, angles and torsion angles

Fractional atomic coordinates and isotropic or equivalent isotropic displacement parameters $\left(\AA^{2}\right)$

\begin{tabular}{lllll}
\hline & $x$ & $y$ & $z$ & $U_{\text {iso }} * U_{\text {eq }}$ \\
\hline O1 & $0.3420(4)$ & $0.18714(15)$ & $1.00233(9)$ & $0.0714(5)$ \\
O2 & $0.4634(3)$ & $-0.00243(13)$ & $0.85678(9)$ & $0.0648(4)$ \\
C1 & $0.2847(3)$ & $0.24041(16)$ & $0.81364(11)$ & $0.0432(4)$ \\
C2 & $0.3024(3)$ & $0.18897(16)$ & $0.69499(12)$ & $0.0428(4)$ \\
C3 & $0.2316(3)$ & $0.29147(16)$ & $0.61941(12)$ & $0.0434(4)$ \\
C4 & $0.1418(4)$ & $0.44533(18)$ & $0.66466(14)$ & $0.0509(5)$ \\
C5 & $0.1251(4)$ & $0.49579(18)$ & $0.78270(14)$ & $0.0558(5)$ \\
C6 & $0.1965(4)$ & $0.39454(18)$ & $0.85767(13)$ & $0.0509(5)$ \\
C7 & $0.3696(4)$ & $0.13271(17)$ & $0.89319(12)$ & $0.0477(4)$ \\
C8 & $0.2570(4)$ & $0.23974(16)$ & $0.49657(12)$ & $0.0489(5)$ \\
C9 & $0.2828(5)$ & $0.1985(2)$ & $0.39633(14)$ & $0.0629(6)$ \\
H1 & $0.421(7)$ & $0.110(3)$ & $1.054(2)$ & $0.133(9)^{*}$ \\
H2 & $0.362(4)$ & $0.086(2)$ & $0.6622(13)$ & $0.050(4)^{*}$ \\
H4 & $0.088(4)$ & $0.519(2)$ & $0.6115(14)$ & $0.063(4)^{*}$ \\
H5 & $0.058(4)$ & $0.601(2)$ & $0.8124(14)$ & $0.069(5)^{*}$ \\
H6 & $0.188(4)$ & $0.429(2)$ & $0.9398(16)$ & $0.065(5)^{*}$ \\
H9 & $0.302(5)$ & $0.164(2)$ & $0.3144(19)$ & $0.087(6)^{*}$ \\
\end{tabular}

Atomic displacement parameters $\left(\AA^{2}\right)$

\begin{tabular}{lllllll}
\hline & $U^{11}$ & $U^{22}$ & $U^{33}$ & $U^{12}$ & $U^{13}$ & $U^{23}$ \\
\hline O1 & $0.1157(10)$ & $0.0684(7)$ & $0.0382(6)$ & $0.0396(7)$ & $0.0105(6)$ & $0.0108(5)$ \\
O2 & $0.1041(9)$ & $0.0569(7)$ & $0.0434(6)$ & $0.0361(6)$ & $0.0138(5)$ & $0.0146(5)$
\end{tabular}


supporting information

\begin{tabular}{lllllll} 
C1 & $0.0424(7)$ & $0.0445(7)$ & $0.0430(7)$ & $0.0091(5)$ & $0.0044(5)$ & $0.0095(5)$ \\
C2 & $0.0462(8)$ & $0.0391(7)$ & $0.0442(7)$ & $0.0111(5)$ & $0.0051(5)$ & $0.0090(5)$ \\
C3 & $0.0393(7)$ & $0.0462(7)$ & $0.0459(7)$ & $0.0082(5)$ & $0.0036(5)$ & $0.0129(5)$ \\
C4 & $0.0517(8)$ & $0.0463(8)$ & $0.0580(9)$ & $0.0138(6)$ & $0.0011(6)$ & $0.0165(6)$ \\
C5 & $0.0599(9)$ & $0.0448(8)$ & $0.0634(10)$ & $0.0201(6)$ & $0.0027(7)$ & $0.0060(7)$ \\
C6 & $0.0524(8)$ & $0.0512(8)$ & $0.0484(8)$ & $0.0155(6)$ & $0.0052(6)$ & $0.0041(6)$ \\
C7 & $0.0566(8)$ & $0.0479(7)$ & $0.0399(7)$ & $0.0139(6)$ & $0.0064(6)$ & $0.0084(6)$ \\
C8 & $0.0526(8)$ & $0.0485(8)$ & $0.0510(9)$ & $0.0148(6)$ & $0.0052(6)$ & $0.0189(6)$ \\
C9 & $0.0827(12)$ & $0.0647(10)$ & $0.0483(9)$ & $0.0250(8)$ & $0.0116(8)$ & $0.0176(7)$ \\
\hline
\end{tabular}

Geometric parameters $\left(\AA,{ }^{o}\right)$

\begin{tabular}{|c|c|c|c|}
\hline $\mathrm{O} 1-\mathrm{C} 7$ & $1.2902(18)$ & $\mathrm{C} 4-\mathrm{C} 5$ & $1.376(2)$ \\
\hline $\mathrm{O} 2-\mathrm{C} 7$ & $1.2415(18)$ & $\mathrm{C} 5-\mathrm{C} 6$ & $1.378(2)$ \\
\hline $\mathrm{O} 1-\mathrm{H} 1$ & $1.03(2)$ & $\mathrm{C} 8-\mathrm{C} 9$ & $1.175(2)$ \\
\hline $\mathrm{C} 1-\mathrm{C} 2$ & $1.3845(19)$ & $\mathrm{C} 2-\mathrm{H} 2$ & $0.938(16)$ \\
\hline $\mathrm{C} 1-\mathrm{C} 6$ & $1.389(2)$ & $\mathrm{C} 4-\mathrm{H} 4$ & $0.992(16)$ \\
\hline $\mathrm{C} 1-\mathrm{C} 7$ & $1.4735(19)$ & $\mathrm{C} 5-\mathrm{H} 5$ & $0.959(17)$ \\
\hline $\mathrm{C} 2-\mathrm{C} 3$ & $1.3907(19)$ & $\mathrm{C} 6-\mathrm{H} 6$ & $0.955(18)$ \\
\hline $\mathrm{C} 3-\mathrm{C} 4$ & $1.393(2)$ & C9- $\mathrm{H} 9$ & $0.96(2)$ \\
\hline $\mathrm{C} 3-\mathrm{C} 8$ & $1.437(2)$ & & \\
\hline $\mathrm{C} 7-\mathrm{O} 1-\mathrm{H} 1$ & $112.4(13)$ & $\mathrm{O} 2-\mathrm{C} 7-\mathrm{C} 1$ & $121.61(12)$ \\
\hline $\mathrm{C} 2-\mathrm{C} 1-\mathrm{C} 7$ & $119.56(12)$ & $\mathrm{O} 1-\mathrm{C} 7-\mathrm{C} 1$ & $116.24(13)$ \\
\hline $\mathrm{C} 6-\mathrm{C} 1-\mathrm{C} 7$ & $120.24(12)$ & $\mathrm{C} 3-\mathrm{C} 8-\mathrm{C} 9$ & $179.04(17)$ \\
\hline $\mathrm{C} 2-\mathrm{C} 1-\mathrm{C} 6$ & $120.17(12)$ & $\mathrm{C} 1-\mathrm{C} 2-\mathrm{H} 2$ & $122.5(9)$ \\
\hline $\mathrm{C} 1-\mathrm{C} 2-\mathrm{C} 3$ & $120.08(12)$ & $\mathrm{C} 3-\mathrm{C} 2-\mathrm{H} 2$ & $117.4(9)$ \\
\hline $\mathrm{C} 2-\mathrm{C} 3-\mathrm{C} 8$ & $120.12(12)$ & $\mathrm{C} 3-\mathrm{C} 4-\mathrm{H} 4$ & $119.9(9)$ \\
\hline $\mathrm{C} 4-\mathrm{C} 3-\mathrm{C} 8$ & $120.72(13)$ & $\mathrm{C} 5-\mathrm{C} 4-\mathrm{H} 4$ & $119.6(9)$ \\
\hline $\mathrm{C} 2-\mathrm{C} 3-\mathrm{C} 4$ & $119.15(13)$ & $\mathrm{C} 4-\mathrm{C} 5-\mathrm{H} 5$ & $119.5(10)$ \\
\hline $\mathrm{C} 3-\mathrm{C} 4-\mathrm{C} 5$ & $120.48(14)$ & $\mathrm{C} 6-\mathrm{C} 5-\mathrm{H} 5$ & $120.1(10)$ \\
\hline $\mathrm{C} 4-\mathrm{C} 5-\mathrm{C} 6$ & $120.39(14)$ & $\mathrm{C} 1-\mathrm{C} 6-\mathrm{H} 6$ & $119.3(10)$ \\
\hline $\mathrm{C} 1-\mathrm{C} 6-\mathrm{C} 5$ & $119.73(14)$ & $\mathrm{C} 5-\mathrm{C} 6-\mathrm{H} 6$ & $121.0(10)$ \\
\hline $\mathrm{O} 1-\mathrm{C} 7-\mathrm{O} 2$ & $122.16(13)$ & $\mathrm{C} 8-\mathrm{C} 9-\mathrm{H} 9$ & 179.5 (19) \\
\hline $\mathrm{C} 6-\mathrm{C} 1-\mathrm{C} 2-\mathrm{C} 3$ & $-0.05(19)$ & $\mathrm{C} 6-\mathrm{C} 1-\mathrm{C} 7-\mathrm{O} 2$ & $-176.98(14)$ \\
\hline $\mathrm{C} 7-\mathrm{C} 1-\mathrm{C} 2-\mathrm{C} 3$ & $-178.37(12)$ & $\mathrm{C} 1-\mathrm{C} 2-\mathrm{C} 3-\mathrm{C} 4$ & $-0.26(19)$ \\
\hline $\mathrm{C} 2-\mathrm{C} 1-\mathrm{C} 6-\mathrm{C} 5$ & $0.3(2)$ & $\mathrm{C} 1-\mathrm{C} 2-\mathrm{C} 3-\mathrm{C} 8$ & $178.71(12)$ \\
\hline $\mathrm{C} 7-\mathrm{C} 1-\mathrm{C} 6-\mathrm{C} 5$ & $178.59(14)$ & $\mathrm{C} 2-\mathrm{C} 3-\mathrm{C} 4-\mathrm{C} 5$ & $0.3(2)$ \\
\hline $\mathrm{C} 2-\mathrm{C} 1-\mathrm{C} 7-\mathrm{O} 1$ & $-178.90(13)$ & $\mathrm{C} 8-\mathrm{C} 3-\mathrm{C} 4-\mathrm{C} 5$ & $-178.63(14)$ \\
\hline $\mathrm{C} 2-\mathrm{C} 1-\mathrm{C} 7-\mathrm{O} 2$ & $1.3(2)$ & $\mathrm{C} 3-\mathrm{C} 4-\mathrm{C} 5-\mathrm{C} 6$ & $-0.1(2)$ \\
\hline $\mathrm{C} 6-\mathrm{C} 1-\mathrm{C} 7-\mathrm{O} 1$ & $2.8(2)$ & $\mathrm{C} 4-\mathrm{C} 5-\mathrm{C} 6-\mathrm{C} 1$ & $-0.2(2)$ \\
\hline
\end{tabular}

Hydrogen-bond geometry $\left(\AA,{ }^{\circ}\right)$

\begin{tabular}{lllll}
\hline$D-\mathrm{H}^{\prime} \cdots A$ & $D-\mathrm{H}$ & $\mathrm{H} \cdots A$ & $D \cdots A$ & $D-\mathrm{H} \cdots A$ \\
\hline $\mathrm{O} 1-\mathrm{H} 1 \cdots \mathrm{O} 2^{\mathrm{i}}$ & $1.03(2)$ & $1.59(2)$ & $2.625(2)$ & $175(2)$
\end{tabular}


$\mathrm{C} 9-\mathrm{H} 9 \cdots \mathrm{O} 2^{\mathrm{ii}}$

$0.96(2)$

Symmetry codes: (i) $-x+1,-y,-z+2$; (ii) $-x+1,-y,-z+1$.
2.50 (2)

$3.386(2)$

$153(2)$ 